\title{
IMPACT OF AWARENESS PROGRAMS ON CHOLERA DYNAMICS: TWO MODELING APPROACHES
}

By

Chayu Yang

Jin Wang

Professor of Mathematics (Chair)

Roger Nichols

Associate Professor of Mathematics (Committee member)
Lingju Kong

Professor of Mathematics

(Committee member)

Feng Bao

Associate Professor of Mathematics (Committee member) 


\title{
IMPACT OF AWARENESS PROGRAMS ON CHOLERA DYNAMICS: TWO MODELING APPROACHES
}

\author{
By \\ Chayu Yang \\ A Thesis Submitted to the Faculty of the University of \\ Tennessee at Chattanooga in Partial \\ Fulfillment of the Requirements of the Degree \\ of Master of Science: Mathematics
}

The University of Tennessee at Chattanooga

Chattanooga, Tennessee

August 2017 


\begin{abstract}
We propose two differential equation-based models to investigate the impact of awareness programs on cholera dynamics. The first model represents the disease transmission rates as decreasing functions of the number of awareness programs, whereas the second model divides the susceptible individuals into two distinct classes depending on their awareness/unawareness of the risk of infection. We study the essential dynamical properties of each model, using both analytical and numerical approaches. We find that the two models, though closely related, exhibit significantly different dynamical behaviors. Namely, the first model follows regular threshold dynamics while rich dynamical behaviors such as backward bifurcation may arise from the second one. Our results highlight the importance of validating key modeling assumptions in the development and selection of mathematical models toward practical application.
\end{abstract}




\section{DEDICATION}

This thesis is dedicated to my wife Meng Tang, mother Wenliang Tang and father Yuantong Yang. They always give me enough support and endless love. 


\section{ACKNOWLEDGEMENT}

I am grateful to the Department of Mathematics of the University of Tennessee at Chattanooga for the support it always provides to me. I cannot express enough thanks to my advisor Dr. Jin Wang for his continued concern and guidance throughout the research process. I offer my sincere appreciation for the learning opportunities provided by my advisor. 


\section{TABLE OF CONTENTS}

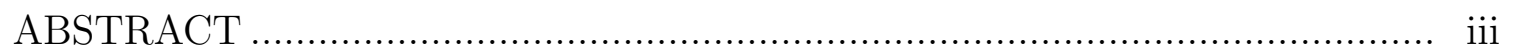

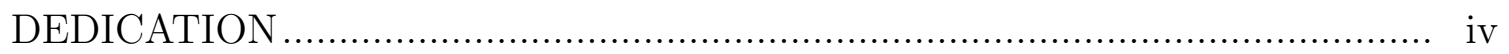

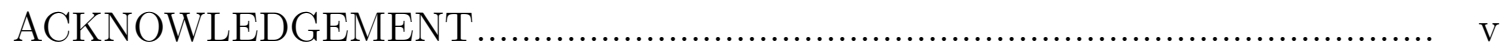

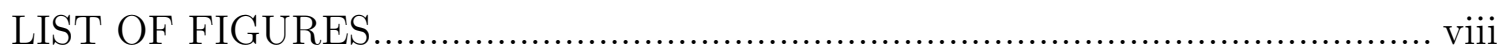

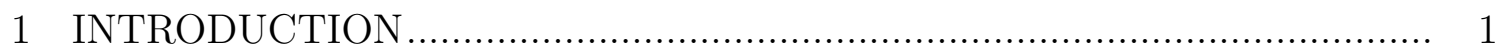

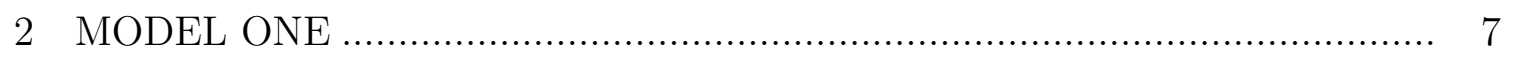

2.1 Model formulation........................................................................ 7

2.2 Basic reproduction number .......................................................... 8

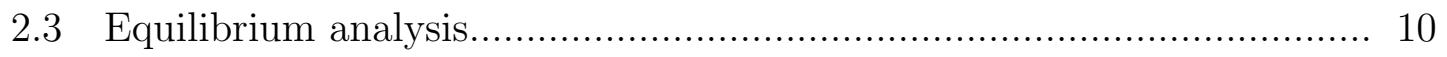

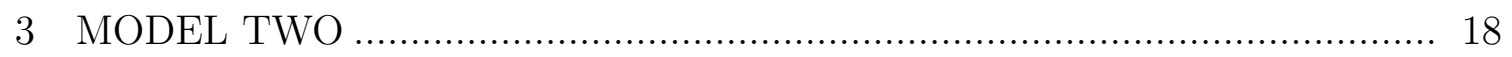

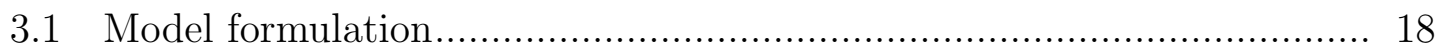

3.2 Basic reproduction number ........................................................... 19

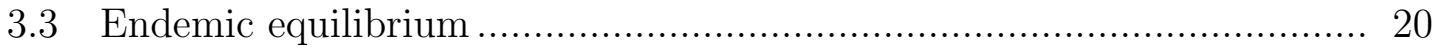

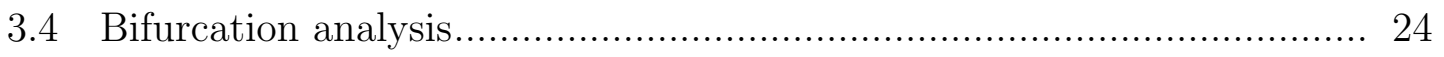

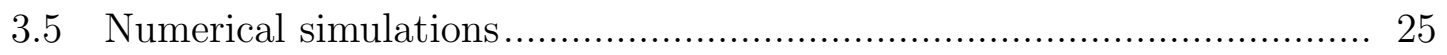

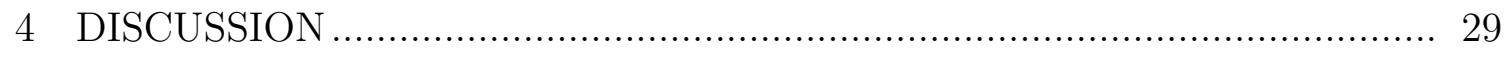

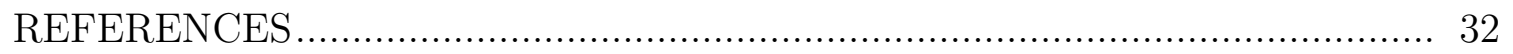




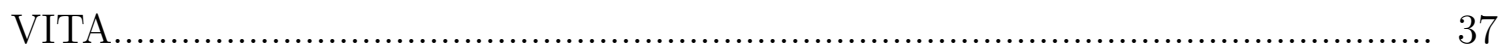




\section{LIST OF FIGURES}

3.1 Forward and backward bifurcation . . . . . . . . . . . . . . 26

3.2 The number of infectious humans decays along time . . . . . . . . 26

3.3 Phase portrait for $\mathcal{R}_{0}=0.976 \ldots \ldots$. . . . . . . . . . 27

3.4 Phase portrait for $\mathcal{R}_{0}=1.4 \ldots \ldots \ldots \ldots$. . . . . . . . . 28 


\section{CHAPTER 1}

\section{INTRODUCTION}

Over the last decade a number of major cholera outbreaks took place that spanned the continents of Africa, America, and Asia, indicating that cholera, an ancient disease, has re-emerged as a serious public health threat worldwide. Cholera is an acute diarrhoeal disease that can spread rapidly in populations with limited access to clean water and sanitation resources, especially in areas where the public health infrastructure is underdeveloped. The causing agent for human cholera is the bacterium Vibrio cholerae, either type $\mathrm{O} 1$ or O139, which can be transmitted both from the contaminated aquatic environment, and from human-to-human contacts such as shaking hands or eating food prepared by infected individuals [15,24]. About 5\% of those who are infected develop severe symptoms including acute watery diarrhea, vomiting, and leg cramps. Experimental studies show that the fecal shedding from infected individuals contain large doses of vibrios, and these freshly shed vibrios have a much higher infectivity (up to 700-fold) than that of the vibrios in the environment [10,25].

Several control measures have been recommended in the prevention and control of cholera epidemics. Investment in water and sanitation is the key for long-term cholera control, but it is usually not possible to establish or to maintain necessary hygienic facilities in epidemic and emergency settings. Basic oral rehydration therapy using drinking water with modest amounts of sugar and salt added is credited for saving a huge number of lives and reducing the case fatality rate of cholera below 1\% [14]. Oral cholera vaccines have been successfully deployed in protecting populations at 
high risk of cholera, and there has been recently renewed interest in mass vaccination under outbreak and emergency settings [21,22]. In addition, severe cholera patients are treated with antibiotics capable of reducing the duration and severity of illness, though mass administration of antibiotics is not recommended due to the risk of increasing antimicrobial resistance [18]. Many theoretical and clinical studies have been conducted for cholera and its prevention, treatment, and control strategies (a recent review article [25] and references therein). In particular, a number of mathematical models have been proposed and analyzed to understand the disease mechanism, to assess the utility of various control measures, and to predict the occurrence of cholera outbreaks and trends (see, e.g., $[2,3,15,24,26,28,31,32,35,36])$.

Non-pharmaceutical interventions such as behavioral change and waste management, on the other hand, play an important role in reducing exposure to infectious agents and hence the disease morbidity and mortality and in shaping the epidemic and endemic patterns. For example, people who are conscious of the infection risk will naturally avoid or reduce contacts with infected individuals and ingestion of contaminated water or food in order to protect themselves and their family members. In case of serious outbreaks, people who have known basic facts about a disease (e.g., transmission modes and symptoms) will attempt to adjust their routine schedules in work, travel and recreation-related activities, to pay more attention to sanitation and hygiene practice (e.g., washing hands often with soap, properly treating disposals from infected individuals), and to receive vaccination or antibiotic prophylaxis, so that their risk of infection could be reduced. Thus, human behavior can contribute significantly toward the control and possible eradication of an infectious disease, cholera in particular, and any efforts motivating such positive changes of human behavior should be promoted. 
Creation of disease awareness programs, among others, can be an effective approach to educate the general public on the infection risk and outbreak severity, and to convince people to make necessary changes of their routine behavior so as to reduce the exposure to the causative agent and the possibility of infection. Such programs can be implemented either physically (with health professionals on site), or through news media including fliers, posters, newspapers, television and radio advertisements, with the common goals of communicating basic knowledge of the disease to the public and directing people toward appropriate prevention and intervention during a disease outbreak. The awareness programs for cholera would be especially useful, since epidemic and endemic cholera occur primarily in countries and regions where poverty prevails and where people have very limited access to internet and social media that have been increasingly common in developed countries as the main resource for information update [9].

Following the pioneering work of Capasso and Serio [4], Liu et al. [20], and Cui et al. [7], there have been quite a few studies on the mathematical modeling of human behavior during disease outbreaks [5, 12, 13, 16, 17, 23, 29, 33, 37-39]. Among these, we would like to mention those with an explicit assessment of the effect of awareness programs. Misra et al. [23] presented a nonlinear mathematical model that incorporated awareness programs, driven by the disease prevalence, and their analysis showed that the spread of infection diseases can be decreased through media campaigns but the disease remains endemic. Samanta et al. [29] extended the study of Misra et al. [23] by considering that aware susceptible are also vulnerable to disease infection with lower transmission rate than that of unaware susceptible. Kaur et al. [16] modeled and analyzed the effects of awareness programs in reducing disease transmission and spread, using an SIRS (susceptible-infected-recovered-susceptible) 
compartment system. Yorke and London [38] published an SIS compartmental model with the assumption that only a portion of the entire population respond to the disease outbreak and limit their contacts with the infectives. Zuo and Liu [39] proposed an epidemic model with time delay to study awareness programs, and they found that increasing the program implementation rate and awareness dissemination rate can reduce the disease prevalence.

Our main objective in the present paper is to use mathematical modeling, analysis and simulation to assess the effect of awareness programs in the course of a cholera outbreak, which has not been investigated in prior studies. To that end, we incorporate the number of awareness programs as an additional compartment into the cholera modeling framework that consists of the human hosts and the environmental pathogen (i.e., the vibrios). Thus, the awareness programs are explicitly accounted for in our study. Then the central question becomes: how to represent the impact of awareness programs in our cholera model?

We propose two different, but closely related, approaches to model the impact of awareness programs on cholera dynamics. With the presence of awareness programs, people will gain more information regarding the disease outbreak and will likely adjust their routine behavior to avoid or reduce contacts with infected individuals and/or contaminated environments, leading to lower disease transmission and spread. Thus, in our first modeling approach, the effects of the awareness programs are reflected by the change of the transmission rates and host shedding rate in the model. We treat each transmission coefficient as a decreasing function of the number of the awareness programs, and investigate how the varied transmission rates change the epidemics and endemics of cholera. It is somewhat similar to a previous study where transmission rates of the model directly depend on the number of infectives [37]. 
In reality, awareness programs, like any other educational efforts, are limited by available resources and many other socioeconomic factors, and it is usually impossible for these programs to reach the entire host population. As a result, people who are involved in the awareness programs will be more informed about the spread and severity of the disease and will more likely to make efforts to keep from catching the disease. In contrast, those individuals who are not reached by the awareness programs will likely lack knowledge of the disease and will possibly make no change of their daily life, so that they will be at higher risk for contracting the disease. Thus, our second modeling approach aims to represent such a limitation of the awareness programs. We divide the susceptible populations into two classes: one for those with disease awareness, and the other for those without awareness. Individuals in these two classes will have different transmission rates, and may switch to each other: unaware individuals may become conscious of the disease through the interaction with the awareness programs, and aware individuals may lose disease awareness after a certain period of time.

We will first present and analyze Model One, where disease transmission rates and bacterial shedding rate decrease with the number of awareness programs. We will study the disease-free equilibrium of the system and its global asymptotic stability when the basic reproduction number is lower than or equal to unity. We will also establish the existence and uniqueness of the endemic equilibrium, and its global stability, when the basic reproduction number is higher than one. With two separate susceptible classes and their different interaction with infected human hosts, environmental pathogen, and awareness programs, Model Two is more challenging to analyze. We will combine mathematical analysis and numerical simulation to gain insight into the dynamics of Model Two. In particular, we will establish that under 
certain conditions, Model Two exhibits a backward bifurcation, a significant difference from the dynamics of Model One. For the organization of this paper, Sections 2 and 3 are devoted to Models One and Two, respectively. Section 4 concludes the paper with discussion on the findings, implications, and limitations of this work. 


\section{CHAPTER 2}

\section{MODEL ONE}

\subsection{Model formulation}

We start by presenting our first cholera model with the consideration of the impact of awareness programs. Let $S, I$ and $R$ represent the number of susceptible, infectious and recovered human individuals, respectively, and $N=S+I+R$ the total population size. Also let $B$ denote the concentration of $V$. cholerae in the aquatic environment, and $M$ the number of the awareness programs driven by disease prevalence and media

coverage. Susceptible individuals are infected through either human-to-human or environment-to-human route. Infected individuals will recover after a certain period of time, and during the course of infection they contribute (e.g., through shedding) to the growth of vibrios in the environment. Further, we assume that the environmentto-human transmission is subject to a saturation effect of the bacterial concentration. Thus, we use the following differential equations to describe the change of these variables with respect to time:

$$
\begin{aligned}
\frac{d S}{d t} & =\mu N-\beta_{1}(M) S I-\beta_{2}(M) S \frac{B}{B+K}-\mu S+\sigma R \\
\frac{d I}{d t} & =\beta_{1}(M) S I+\beta_{2}(M) S \frac{B}{B+K}-(\gamma+\mu) I \\
\frac{d R}{d t} & =\gamma I-(\mu+\sigma) R \\
\frac{d B}{d t} & =\xi(M) I-\delta B \\
\frac{d M}{d t} & =\Lambda+\eta I-\nu M .
\end{aligned}
$$


Here $\mu$ is the natural birth and death rate for the human hosts, $K$ is the half saturation concentration of the vibrios, $\sigma$ is the immunity waning rate, $\gamma$ is the rate of recovery from cholera infection, and $\delta$ is the removal rate of vibrios from the environment. The number of awareness programs grows with an influx $\Lambda$. Meanwhile, it is stimulated by the disease prevalence at a rate $\eta$, and decays with time at a rate $\nu$. The parameters $\beta_{1}$ and $\beta_{2}$ are the direct (or, human-to-human) and indirect (or, environment-to-human) transmission rates, respectively, and $\xi$ is the rate of human contribution to the environmental vibrios. We assume that $\beta_{1}, \beta_{2}$ and $\xi$ are all explicitly depending on $M$ and decrease with the growth of $M$, reflecting the impact of the awareness programs on disease transmission and waste management. Specifically, we make the following assumptions on these three media-dependent parameters:

(H1) $\beta_{1}(M), \beta_{2}(M)$ and $\xi(M)$ are positive functions on $\left[0, M_{\max }\right]$;

(H2) $\beta_{1}(M), \beta_{2}(M), \xi(M) \in C^{1}\left(\left[0, M_{\max }\right]\right)$, and $\beta_{1}^{\prime}(M) \leq 0, \beta_{2}^{\prime}(M) \leq 0, \xi^{\prime}(M) \leq 0$ where $M_{\max }=(\Lambda+\eta N) / v$ is an upper bound of $M$.

\subsection{Basic reproduction number}

We will make use of the basic reproduction number [1], denoted as $\mathcal{R}_{0}$, in the analysis of our model (2.1.1). Let us first determine $\mathcal{R}_{0}$ by the standard next generation matrix technique $[8,34]$.

Clearly, the system (2.1.1) always has a unique disease-free equilibrium (DFE) at

$$
E^{0}=\left(S^{0}, I^{0}, R^{0}, B^{0}, M^{0}\right)=(N, 0,0,0, \Lambda / \nu)
$$

Since the infection components in this model are $I$ and $B$, we find that the new 
infection matrix $F$ and the transition matrix $V$ are given by

$$
F=\left[\begin{array}{cc}
b_{1} N & b_{2} N / K \\
0 & 0
\end{array}\right] \text { and } V=\left[\begin{array}{cc}
\gamma+\mu & 0 \\
-b_{3} & \delta
\end{array}\right]
$$

with $b_{1}=\beta_{1}\left(M^{0}\right), b_{2}=\beta_{2}\left(M^{0}\right)$ and $b_{3}=\xi\left(M^{0}\right)$. It follows that the next generation matrix is given by

$$
F V^{-1}=\left[\begin{array}{cc}
\frac{b_{1} N}{\gamma+\mu}+\frac{b_{2} b_{3} N}{\delta K(\gamma+\mu)} & \frac{b_{2} N}{\delta K} \\
0 & 0
\end{array}\right] .
$$

The basic reproduction number of model (2.1.1) is then defined as the spectral radius of the matrix $F V^{-1}$, and we find that

$$
\mathcal{R}_{0}=\rho\left(F V^{-1}\right)=\frac{b_{1} N}{\gamma+\mu}+\frac{b_{2} b_{3} N}{\delta K(\gamma+\mu)}
$$

which provides a measurement for the disease risk during a cholera outbreak. The first term in $\mathcal{R}_{0}$ comes from the direct transmission route, and the second term represents the contribution from the indirect transmission route. It is worthwhile to compare this result with the basic reproduction number when the awareness programs are not present and the transmission rates $\beta_{1}, \beta_{2}$ and shedding rate $\xi$ remain constant; i.e., $\beta_{1}(M)=\beta_{1}(0), \beta_{2}(M)=\beta_{2}(0)$, and $\xi(M)=\xi(0)$. In that case, we have (see, e.g., [24])

$$
\widetilde{\mathcal{R}}_{0}=\frac{\beta_{1}(0) N}{\gamma+\mu}+\frac{\beta_{2}(0) \beta_{3}(0) N}{\delta K(\gamma+\mu)}
$$

Based on assumption (H2), it is straightforward to observe that $R_{0} \leq \widetilde{\mathcal{R}}_{0}$, indicating that the presence of the awareness programs leads to a smaller disease risk. 


\subsection{Equilibrium analysis}

We now analyze the equilibria of the system (2.1.1) which will provide essential information regarding the long-term dynamics of the disease. Let $(S, I, R, B, M)$ be an equilibrium of model (2.1.1), which satisfies the following equations

$$
\begin{aligned}
& \mu N-\beta_{1}(M) S I-\beta_{2}(M) S \frac{B}{B+K}-\mu S+\sigma R=0, \\
& \beta_{1}(M) S I+\beta_{2}(M) S \frac{B}{B+K}-(\gamma+\mu) I=0, \\
& \gamma I-(\mu+\sigma) R=0, \\
& \xi(M) I-\delta B=0, \\
& \Lambda+\eta I-\nu M=0 .
\end{aligned}
$$

Solving (2.3.1) yields

$$
\begin{aligned}
S & =\frac{(\gamma+\mu) I}{\beta_{1}(M) I+\beta_{2}(M) B /(B+K)}, \\
M & =\frac{\Lambda+\eta I}{\nu}, \\
R & =\frac{\gamma}{\mu+\sigma} I \\
B & =\frac{\xi(M)}{\delta} I .
\end{aligned}
$$

It follows from $S+I+R=N$ that the third equation of (2.3.2) implies

$$
S=N-a I=: \phi(I) \quad \text { with } a=1+\gamma /(\mu+\sigma)
$$

Meanwhile, in view of the first equation of (2.3.2), we obtain

$$
S=\frac{\gamma+\mu}{h(I)}=: \psi(I),
$$


where

$$
h(I)=\beta_{1}(\chi(I))+\frac{\beta_{2}(\chi(I)) \xi(\chi(I))}{\xi(\chi(I)) I+\delta K} \quad \text { with } \chi(I)=\frac{\Lambda+\eta I}{\nu} .
$$

Let us now consider curves $S=\phi(I)$ and $S=\psi(I)$. In particular, the intersections of these two curves in $\mathbb{R}_{+}^{2}$ determine the non-DFE equilibria. Note that

$$
\begin{aligned}
h^{\prime}(I)= & \beta_{1}^{\prime}(\chi(I)) \chi^{\prime}(I)+\frac{\beta_{2}^{\prime}(\chi(I)) \chi^{\prime}(I) \xi(\chi(I))}{\xi(\chi(I)) I+\delta K} \\
& +\beta_{2}(\chi(I)) \frac{\delta K \xi^{\prime}(\chi(I)) \chi^{\prime}(I)-\xi^{2}(\chi(I))}{(\xi(\chi(I)) I+\delta K)^{2}}
\end{aligned}
$$

Using assumption (H2) and the fact $\chi^{\prime}(I)=\eta / \nu>0$, we see that $h^{\prime}(I) \leq 0$. This implies that $\psi(I)$ is an increasing function. In contrast, $\phi(I)$ is strictly decreasing. Additionally, one can easily verify that $\psi(0)=N / \mathcal{R}_{0}, \phi(0)=N, \psi(N / a)>0$ and $\phi(N / a)=0$. Hence, we conclude:

(1) If $\mathcal{R}_{0}>1$, these two curves have a unique intersection lying in the interior of $\mathbb{R}_{+}^{2}$, due to $\psi(0)<\phi(0)$ and $\psi(N / a)>\phi(N / a)$; furthermore, at this intersection point, equation (2.3.2) yields $M, R, B>0$ (since $I>0$ ).

(2) If $\mathcal{R}_{0} \leq 1$, the two curves have no intersection in the interior of $\mathbb{R}_{+}^{2}$ as $\psi(0) \geq$ $\phi(0)$.

Therefore, by equation (2.3.2), we find that the model (2.1.1) admits a unique equilibrium, the DFE, if $\mathcal{R}_{0} \leq 1$; and it admits two equilibria, the DFE and an endemic equilibrium (EE), if $\mathcal{R}_{0}>1$.

In what follows, we perform a study on the global stability of the DFE. By a simple comparison principle, we find that $0 \leq B \leq B_{\max }$ and $M^{0} \leq M \leq M_{\max }$, where $B_{\max }=\xi(0) N / \delta$, and $M_{\max }$ is defined in (H2). Thus, it leads to a biological 
feasible domain

$$
\Omega=\left\{(S, I, R, B, M) \in \mathbb{R}_{+}^{5}: S+I+R=N, 0 \leq B \leq B_{\max }, M^{0} \leq M \leq M_{\max }\right\} .
$$

Theorem 1. The following statements hold for the model (2.1.1).

(1) If $\mathcal{R}_{0} \leq 1$, the DFE of system (2.1.1) is globally asymptotically stable in $\Omega$.

(2) If $\mathcal{R}_{0}>1$, the DFE of system (2.1.1) is unstable and there exists a unique endemic equilibrium. Moreover, the disease is uniformly persistent in the interior of $\Omega$, denoted by $\stackrel{\Omega}{\Omega}$, namely, $\liminf _{t \rightarrow \infty}(I(t), B(t))>(c, c)$ for some $c>0$.

Proof. Let $\mathbf{x}=(I, B)^{T}$. One can verify that

$$
\frac{d \mathbf{x}}{d t} \leq(F-V) \mathbf{x}
$$

where the matrices $F$ and $V$ are given in equation (2.2.2). Take $\mathbf{u}=\left(b_{1} N, b_{2} N / K\right)$. It then follows from the fact $\mathcal{R}_{0}=\rho\left(F V^{-1}\right)=\rho\left(V^{-1} F\right)$ and direct calculation that $\mathbf{u}$ is a left eigenvector associated with the eigenvalue $\mathcal{R}_{0}$ of the matrix $V^{-1} F$; i.e., $\mathbf{u} V^{-1} F=\mathcal{R}_{0} \mathbf{u}$. Let us consider a Lyapunov function

$$
\mathcal{L}=\mathbf{u} V^{-1} \mathbf{x}
$$

Differentiating $\mathcal{L}$ along the solutions of (2.1.1), we have

$$
\mathcal{L}^{\prime}=\mathbf{u} V^{-1} \mathbf{x}^{\prime} \leq \mathbf{u} V^{-1}(F-V) \mathbf{x}=\mathbf{u}\left(\mathcal{R}_{0}-1\right) \mathbf{x}
$$

Case 1: $\mathcal{R}_{0}<1$ The equality $\mathcal{L}^{\prime}=0$ implies that $\mathbf{u x}=0$. This leads to $I=B=0$ by noting the positive components of $\mathbf{u}$. Hence, when $\mathcal{R}_{0}<1$, equations of $(2.1 .1)$ 
yield $S=S^{0}, M=M^{0}$ and $I=R=B=0$. Therefore, the invariant set on which $\mathcal{L}^{\prime}=0$ contains only one point which is the DFE.

Case 2: $\mathcal{R}_{0}=1 \quad$ The equality $\mathcal{L}^{\prime}=0$ implies that $\beta_{1}(M) S I=b_{1} N I, \beta_{2}(M) S B /(B+$ $K)=b_{2} N B / K$ and $\xi(M) I=b_{3} I$. Thus, either $I=B=0$, or $B=0, S=N$ and $\beta_{1}(M)=b_{1}$ and $\xi(M)=b_{3}$ holds. The former can proceed as above. Suppose the latter holds, then $\frac{d B}{d t}=\xi(M) I \equiv 0$ which implies $I=0$. Once again this can proceed as before.

Therefore, in either case, the largest invariant set on which $\mathcal{L}^{\prime}=0$ consists of the singleton $E^{0}=\left(N, 0,0,0, M^{0}\right)$. By LaSalle's Invariant Principle [19], the DFE is globally asymptotically stable in $\Omega$ if $\mathcal{R}_{0} \leq 1$.

In contrast, if $\mathcal{R}_{0}>1$, then it follows from the continuity of vector fields that $\mathcal{L}^{\prime}>0$ in a neighborhood of the DFE in $\Omega$. Thus the DFE is unstable by the Lyapunov stability theory.

The last part can be proved by the persistent theory [30] which is similar to the proof of Theorem 2.5 in Gao and Ruan [13].

Remark. Let $\beta_{i}(M)=\beta_{i 0}-\beta_{i 1} f_{i}(M)$ for $i=1,2$, and $\xi(M)=\beta_{30}-\beta_{31} f_{3}(M)$ where $\beta_{i 0}>\beta_{i 1} \geq 0,0 \leq f_{i}(M) \leq 1$ and $f_{i}^{\prime}(M) \geq 0$ for $i=1,2,3$. Similar to Proposition 3.5 in [37], we can show that $\frac{\partial I_{3}^{*}}{\partial \eta}<0$ and $\frac{\partial I_{3}^{*}}{\partial \beta_{i 1}}<0$ for $i=1,2,3$ whenever $\mathcal{R}_{0}>1$. That is, awareness programs can help to reduce the prevalence of cholera.

In addition, we have conducted an analysis on the global asymptotic stability of the endemic equilibrium, and the details are in the following. Essentially, these stability results establish $\mathcal{R}_{0}=1$ as a forward transcritical bifurcation point, or, a sharp threshold for disease dynamics, and indicate that reducing $\mathcal{R}_{0}$ to values at or below unity will be sufficient to eradicate the disease. In other words, the cholera model (2.1.1) exhibits regular threshold dynamics. 
Let $E^{*}=\left(S^{*}, I^{*}, R^{*}, B^{*}, M^{*}\right)$ denote an endemic equilibrium (EE) of model (2.1.1). To establish the global stability of $E^{*}$, we make the following assumptions:

$$
\left(1-\frac{\beta_{1}(M) I}{\beta_{1}\left(M^{*}\right) I^{*}}\right)\left(1-\frac{M \beta_{1}\left(M^{*}\right) I^{*}}{M^{*} \beta_{1}(M) I}\right) \geq 0
$$

for $0 \leq I \leq N$ and $M^{0} \leq M \leq M_{\max }$, and

$$
\left(1-\frac{\beta_{2}(M) B /(B+K)}{\beta_{2}\left(M^{*}\right) B^{*} /\left(B^{*}+K\right)}\right)\left(1-\frac{\beta_{2}\left(M^{*}\right) /\left(B^{*}+K\right)}{\beta_{2}(M) /(B+K)}\right) \geq 0
$$

for $M^{0} \leq M \leq M_{\max }$, and $0 \leq B \leq B_{\max }$.

Theorem 2. Suppose that i) assumptions (A.8) and (A.9) are satisfied; ii) $\xi(M) \equiv \xi$ is constant; iii) $\sigma=0$. If $\mathcal{R}_{0}>1$, then system (2.1.1) has a unique endemic equilibrium $E^{*}$ that is globally asymptotically stable in $\Omega$.

Proof. For system (2.1.1), motivated by [32], we consider a Lyapunov function

$$
\begin{aligned}
\mathcal{L}= & c_{1}\left(S-S^{*}-S^{*} \ln \left(\frac{S}{S^{*}}\right)\right)+c_{1}\left(I-I^{*}-I^{*} \ln \left(\frac{I}{I^{*}}\right)\right) \\
& +c_{2}\left(B-B^{*}-B^{*} \ln \left(\frac{B}{B^{*}}\right)\right)+c_{3}\left(M-M^{*}-M^{*} \ln \left(\frac{M}{M^{*}}\right)\right),
\end{aligned}
$$

where $c_{i}>0(i=1,2,3)$ are constants to be determined. It is easy to verify that $\mathcal{L} \geq 0$ for all $S, I, B, M>0$, and $\mathcal{L}=0$ iff $(S, I, B, M)=\left(S^{*}, I^{*}, B^{*}, M^{*}\right)$. Differentiating $\mathcal{L}$ along solutions of (2.1.1) and applying all equations of (2.3.1) 
except the third one, we obtain

$$
\begin{aligned}
\mathcal{L}^{\prime}= & c_{1}\left(1-\frac{S^{*}}{S}\right) S^{\prime}+c_{1}\left(1-\frac{I^{*}}{I}\right) I^{\prime}+c_{2}\left(1-\frac{B^{*}}{B}\right) B^{\prime}+c_{3}\left(1-\frac{M^{*}}{M}\right) M^{\prime} \\
= & c_{1}\left[-\mu S\left(1-\frac{S^{*}}{S}\right)^{2}+\beta_{1}\left(M^{*}\right) S^{*} I^{*}\left(2-\frac{S^{*}}{S}-\frac{I}{I^{*}}-\frac{\beta_{1}(M) S I I^{*}}{\beta_{1}\left(M^{*}\right) S^{*} I^{*} I}+\frac{\beta_{1}(M) I}{\beta_{1}\left(M^{*}\right) I^{*}}\right)\right] \\
& +c_{1} \beta_{2}\left(M^{*}\right) S^{*} \frac{B^{*}}{B^{*}+K}\left(2-\frac{S^{*}}{S}-\frac{I}{I^{*}}-\frac{\beta_{2}(M) S B /(B+K) I^{*}}{\beta_{2}\left(M^{*}\right) S^{*} B^{*} /\left(B^{*}+K\right) I}+\frac{\beta_{2}(M) B /(B+K)}{\beta_{2}\left(M^{*}\right) B^{*} /\left(B^{*}+K\right)}\right) \\
& +c_{2} \xi I^{*}\left(\frac{I}{I^{*}}-\frac{B}{B^{*}}-\frac{B^{*} I}{B I^{*}}+1\right)+c_{3}\left(-\Lambda \frac{M}{M^{*}}\left(1-\frac{M^{*}}{M}\right)^{2}+\eta I^{*}\left(\frac{I}{I^{*}}-\frac{M}{M^{*}}-\frac{M^{*} I}{M I^{*}}+1\right)\right) .
\end{aligned}
$$

Notice that $x-1 \geq \ln (x)$ for any $x>0$, and the equality holds iff $x=1$. Together with (A.8), we find that

$$
\begin{aligned}
& 2-\frac{S^{*}}{S}-\frac{I}{I^{*}}-\frac{\beta_{1}(M) S I I^{*}}{\beta_{1}\left(M^{*}\right) S^{*} I^{*} I}+\frac{\beta_{1}(M) I}{\beta_{1}\left(M^{*}\right) I^{*}} \\
= & -\left(1-\frac{\beta_{1}(M) I}{\beta_{1}\left(M^{*}\right) I^{*}}\right)\left(1-\frac{M \beta_{1}\left(M^{*}\right) I^{*}}{M^{*} \beta_{1}(M) I}\right)+3-\frac{S^{*}}{S} \\
& -\frac{\beta_{1}(M) S I I^{*}}{\beta_{1}\left(M^{*}\right) S^{*} I^{*} I}-\frac{M \beta_{1}\left(M^{*}\right) I^{*}}{M^{*} \beta_{1}(M) I}-\frac{I}{I^{*}}+\frac{M}{M^{*}} \\
\leq & -\left(\frac{S^{*}}{S}-1\right)-\left(\frac{\beta_{1}(M) S I I^{*}}{\beta_{1}\left(M^{*}\right) S^{*} I^{*} I}-1\right)-\left(\frac{M \beta_{1}\left(M^{*}\right) I^{*}}{M^{*} \beta_{1}(M) I}-1\right)-\frac{I}{I^{*}}+\frac{M}{M^{*}} \\
= & -\ln \left(\frac{S^{*}}{S} \frac{\beta_{1}(M) S I I^{*}}{\beta_{1}\left(M^{*}\right) S^{*} I^{*} I} \frac{\left.M^{*}\right) I^{*}}{M^{*} \beta_{1}(M) I}\right)-\frac{I}{I^{*}}+\frac{M}{M^{*}} \\
= & \frac{M}{M^{*}}-\ln \left(\frac{M}{M^{*}}\right)-\frac{I}{I^{*}}+\ln \left(\frac{I}{I^{*}}\right) .
\end{aligned}
$$

Likewise, using (A.9), we obtain

$$
\begin{aligned}
& 2-\frac{S^{*}}{S}-\frac{I}{I^{*}}-\frac{\beta_{2}(M) S B /(B+K) I^{*}}{\beta_{2}\left(M^{*}\right) S^{*} B^{*} /\left(B^{*}+K\right) I}+\frac{\beta_{2}(M) B /(B+K)}{\beta_{2}\left(M^{*}\right) B^{*} /\left(B^{*}+K\right)} \\
\leq & \frac{B}{B^{*}}-\ln \left(\frac{B}{B^{*}}\right)-\frac{I}{I^{*}}+\ln \left(\frac{I}{I^{*}}\right) .
\end{aligned}
$$


Meanwhile, one can verify that

$$
\begin{aligned}
& \frac{I}{I^{*}}-\frac{B}{B^{*}}-\frac{B^{*} I}{B I^{*}}+1=-\left(\frac{B^{*} I}{B I^{*}}-1\right)+\frac{I}{I^{*}}-\frac{B}{B^{*}} \\
\leq & -\ln \left(\frac{B^{*} I}{B I^{*}}\right)+\frac{I}{I^{*}}-\frac{B}{B^{*}}=\frac{I}{I^{*}}-\ln \left(\frac{I}{I^{*}}\right)-\frac{B}{B^{*}}+\ln \left(\frac{B}{B^{*}}\right) .
\end{aligned}
$$

Similarly, we have

$$
\frac{I}{I^{*}}-\frac{M}{M^{*}}-\frac{M^{*} I}{M I^{*}}+1 \leq \frac{I}{I^{*}}-\ln \left(\frac{I}{I^{*}}\right)-\frac{M}{M^{*}}+\ln \left(\frac{M}{M^{*}}\right) .
$$

It follows from (A.11)-(A.14) that the equation (A.10) yields

$$
\begin{aligned}
\mathcal{L}^{\prime} \leq & c_{1} \beta_{1}\left(M^{*}\right) S^{*} I^{*}\left(\frac{M}{M^{*}}-\ln \left(\frac{M}{M^{*}}\right)-\frac{I}{I^{*}}+\ln \left(\frac{I}{I^{*}}\right)\right) \\
& +c_{1} \beta_{2}\left(M^{*}\right) S^{*} \frac{B^{*}}{B^{*}+K}\left(\frac{B}{B^{*}}-\ln \left(\frac{B}{B^{*}}\right)-\frac{I}{I^{*}}+\ln \left(\frac{I}{I^{*}}\right)\right) \\
& +c_{2} \xi I^{*}\left(\frac{I}{I^{*}}-\ln \left(\frac{I}{I^{*}}\right)-\frac{B}{B^{*}}+\ln \left(\frac{B}{B^{*}}\right)\right) \\
& +c_{3} \eta I^{*}\left(\frac{I}{I^{*}}-\ln \left(\frac{I}{I^{*}}\right)-\frac{M}{M^{*}}+\ln \left(\frac{M}{M^{*}}\right)\right) .
\end{aligned}
$$

Take $c_{1}=\xi \eta I^{*}, c_{2}=\eta \beta_{2}\left(M^{*}\right) S^{*} B^{*} /\left(B^{*}+K\right)$ and $c_{3}=\xi \beta_{1}\left(M^{*}\right) S^{*} I^{*}$. One can verify by direct calculation that the right hand side of the inequality (A.15) is zero. This shows $\mathcal{L}^{\prime} \leq 0$ with the chosen positive constants $c_{1}, c_{2}$ and $c_{3}$. Moreover, if $\mathcal{L}^{\prime}=0$, then there exists a constant $\hat{k}$ such that

$$
S=S^{*}, I=\hat{k} I^{*}, \quad B=\hat{k} B^{*}, \quad M=\hat{k} M^{*} .
$$

However, by the last equation of (2.3.1), $0=\Lambda+\eta \hat{k} I^{*}-v \hat{k} M^{*}$. This implies that $\hat{k}=1$. Meanwhile, $R=R^{*}$ which follows from the third equation of (2.3.1). Thus, the largest invariant set for which $\mathcal{L}^{\prime}=0$ contains only the EE. Therefore, by 
LaSalle's Invariant Principle [19], the EE is globally asymptotically stable in $\Omega$ when $\mathcal{R}_{0}>1$ 


\section{CHAPTER 3}

\section{MODEL TWO}

\subsection{Model formulation}

In this section, we may abuse some notations whose meaning should be clear from the context. In our second cholera model, we divide the class of susceptible human individuals into two groups: one for those people who are aware of the disease, denoted by $S_{a}$, and the other for those who are unaware of the disease, denoted by $S_{u}$. Individuals in the $S_{a}$ compartment have lower chances of contracting the disease than those in $S_{u}$. Unaware individuals may switch to the aware group due to the involvement with the awareness programs, and aware individuals may lose the awareness of cholera after a period of time. The model then takes the form:

$$
\begin{aligned}
\frac{d S_{u}}{d t} & =\mu N-\beta_{1} S_{u} I-\beta_{2} S_{u} \frac{B}{B+K}-\varrho S_{u} M-\mu S_{u}+(1-p) \sigma R+\kappa S_{a}, \\
\frac{d I}{d t} & =\beta_{1}\left(S_{u}+\alpha_{1} S_{a}\right) I+\beta_{2}\left(S_{u}+\alpha_{2} S_{a}\right) \frac{B}{B+K}-(\gamma+\mu) I, \\
\frac{d R}{d t} & =\gamma I-(\mu+\sigma) R, \\
\frac{d B}{d t} & =\xi I-\delta B, \\
\frac{d S_{a}}{d t} & =\varrho S_{u} M-\beta_{1} \alpha_{1} S_{a} I-\beta_{2} \alpha_{2} S_{a} \frac{B}{B+K}-\mu S_{a}+p \sigma R-\kappa S_{a}, \\
\frac{d M}{d t} & =\Lambda+\eta I-\nu M,
\end{aligned}
$$

where the total human population size is $N=S_{u}+S_{a}+I+R$. For the unaware compartment $S_{u}$, the direct and indirect transmission rates are represented by $\beta_{1}$ 
and $\beta_{2}$, respectively, which are assumed to be constant at all times. For the aware compartment $S_{a}$, the disease transmission rates are lower and are given by $\beta_{1} \alpha_{1}$ and $\beta_{2} \alpha_{2}$, respectively, where $0 \leq \alpha_{1}, \alpha_{2} \leq 1$. The human contribution rate $\xi$ is also assumed to be constant. Unaware individuals gain knowledge of the disease and enter the $S_{a}$ class through interacting with the awareness programs at a rate $\varrho$. Meanwhile, aware individuals become unaware of the disease over time and enter the $S_{u}$ class at a rate $\kappa$. In addition, recovered individual go back to the $S_{a}$ and $S_{u}$ classes, at the fractions $p$ and $1-p$, respectively. Other variables and parameters have the same meaning as those in the first model.

\subsection{Basic reproduction number}

It is easy to verify that system (3.1.1) has a unique DFE:

$$
E^{0}=\left(S_{u}^{0}, I^{0}, R^{0}, B^{0}, S_{a}^{0}, M^{0}\right)=\left(\frac{(\mu+\kappa)}{\mu+\kappa+\varrho \Lambda / \nu} N, 0,0,0, \frac{\varrho \Lambda / \nu}{\mu+\kappa+\varrho \Lambda / \nu} N, \frac{\Lambda}{\nu}\right)
$$

We find that the new infection matrix $F$ and the transition matrix $V$ for this model are given by:

$$
F=\left[\begin{array}{cc}
\beta_{1}\left(S_{u}^{0}+\alpha_{1} S_{a}^{0}\right) & \beta_{2}\left(S_{u}^{0}+\alpha_{2} S_{a}^{0}\right) / K \\
0 & 0
\end{array}\right] \text { and } V=\left[\begin{array}{cc}
\gamma+\mu & 0 \\
-\xi & \delta
\end{array}\right]
$$

Then the next generation matrix is

$$
F V^{-1}=\frac{1}{\gamma+\mu}\left[\begin{array}{cc}
\beta_{1}\left(S_{u}^{0}+\alpha_{1} S_{a}^{0}\right)+\beta_{2}\left(S_{u}^{0}+\alpha_{2} S_{a}^{0}\right) \frac{\xi}{\delta K} & \beta_{2}\left(S_{u}^{0}+\alpha_{2} S_{a}^{0}\right) \frac{\gamma+\mu}{\delta K} \\
0 & 0
\end{array}\right]
$$


Hence the basic reproduction number $\mathcal{R}_{0}=\rho\left(F V^{-1}\right)$ is found as

$$
\mathcal{R}_{0}=\frac{N}{(\gamma+\mu)\left(\mu+\kappa+\varrho M^{0}\right)}\left(\beta_{1}\left(\mu+\kappa+\alpha_{1} \varrho M^{0}\right)+\beta_{2}\left(\mu+\kappa+\alpha_{2} \varrho M^{0}\right) \frac{\xi}{\delta K}\right) .
$$

Again, we observe that the expression of $\mathcal{R}_{0}$ includes the contributions from both direct and indirect transmission routes. When there are no awareness programs, the formula (3.2.1) is reduced to, by simply setting $\Lambda=0$ or $M^{0}=0$,

$$
\widetilde{\mathcal{R}}_{0}=\frac{N}{\gamma+\mu}\left(\beta_{1}+\beta_{2} \frac{\xi}{\delta K}\right)
$$

which is identical to $(2.2 .5)$, by noting that $\beta_{1}, \beta_{2}$ and $\xi$ correspond to $\beta_{1}(0), \beta_{2}(0)$ and $\xi(0)$, respectively. Comparing $\mathcal{R}_{0}$ and $\widetilde{\mathcal{R}}_{0}$, it is obvious that $\mathcal{R}_{0} \leq \widetilde{\mathcal{R}}_{0}$ since $0<\alpha_{1}, \alpha_{2} \leq 1$, a result that can be naturally expected due to the impact of the awareness programs. Importantly, the basic reproduction of Model Two is always less than or equal to that of Model One with equality if and only if $\alpha_{1}=\alpha_{2}=1$ provided that $\beta_{1}=b_{1}, \beta_{2}=b_{2}$ and $\xi=b_{3}$.

\subsection{Endemic equilibrium}

We proceed to investigate the endemic equilibrium (EE) of model (3.1.1). For simplicity, we denote

$$
\gamma_{\mu}=\gamma+\mu, \quad \sigma_{\mu}=\sigma+\mu, \quad \kappa_{\mu}=\kappa+\mu
$$


The endemic equilibrium satisfies

$$
\begin{aligned}
& N=S_{u}+S_{a}+I+R, \\
& R=\frac{\gamma}{\sigma_{\mu}} I, \\
& B=\frac{\xi}{\delta} I \\
& M=\frac{\Lambda+\eta I}{\nu}, \\
& \left(\beta_{1} I+\frac{\beta_{2} B}{B+K}\right) S_{u}+\left(\beta_{1} \alpha_{1} I+\frac{\beta_{2} \alpha_{2} B}{B+K}\right) S_{a}=\gamma_{\mu} I, \\
& \varrho M S_{u}-\left(\beta_{1} \alpha_{1} I+\frac{\beta_{2} \alpha_{2} B}{B+K}+\kappa_{\mu}\right) S_{a}=-p \sigma R .
\end{aligned}
$$

Since $I>0$, substituting equations (3.3.2)-(3.3.4) into (3.3.5) and (3.3.6) yields

$$
S_{u}+S_{a}=\frac{A_{2} I^{2}+A_{1} I+A_{0} D_{0}}{B_{2} I^{2}+B_{1} I+B_{0} D_{0}+\frac{D_{1} I}{I+D_{0}}},
$$

where

$$
\begin{aligned}
& A_{0}=\gamma_{\mu}\left(\kappa_{\mu}+\varrho \frac{\Lambda}{\nu}\right) \\
& A_{1}=\frac{p \sigma \gamma \beta_{2}\left(1-\alpha_{2}\right)}{\sigma_{\mu}}+\beta_{2} \alpha_{2} \gamma_{\mu}+A_{2} D_{0}+\gamma_{\mu}\left(\kappa_{\mu}+\varrho \frac{\Lambda}{\nu}\right) \\
& A_{2}=\gamma_{\mu}\left(\beta_{1} \alpha_{1}+\varrho \frac{\eta}{\nu}\right)+\frac{p \sigma \gamma \beta_{1}\left(1-\alpha_{1}\right)}{\sigma_{\mu}}, \\
& B_{0}=\beta_{1}\left(\kappa_{\mu}+\alpha_{1} \varrho \frac{\Lambda}{\nu}\right)+\frac{\beta_{2}}{D_{0}}\left(\kappa_{\mu}+\alpha_{2} \varrho \frac{\Lambda}{\nu}\right), \\
& B_{1}=B_{2} D_{0}+\beta_{1}\left(\kappa_{\mu}+\alpha_{1} \varrho \frac{\Lambda}{\nu}\right)+\beta_{2} \alpha_{2}\left(\beta_{1}+\varrho \frac{\eta}{\nu}\right)+\beta_{2} \beta_{1} \alpha_{1}, \\
& B_{2}=\beta_{1} \alpha_{1}\left(\beta_{1}+\varrho \frac{\eta}{\nu}\right) \\
& D_{0}=\frac{\delta K}{\xi} \\
& D_{1}=\beta_{2}^{2} \alpha_{2} .
\end{aligned}
$$


From (3.3.1), we have $S_{u}+S_{a}=N-I-R=N-b I$, where $b=1+\frac{\gamma}{\sigma_{\mu}}$. We denote

$$
f(I)=A_{2} I^{2}+A_{1} I+A_{0} D_{0}
$$

and

$$
g(I)=(N-b I)\left(B_{2} I^{2}+B_{1} I+B_{0} D_{0}+\frac{D_{1} I}{I+D_{0}}\right) .
$$

Then at the endemic equilibrium we have

$$
f(I)=g(I), \quad I \in(0, N / b) .
$$

Since $A_{i}>0(i=0,1,2)$, it is straightforward to see $f^{\prime}(I)>0$ and $f^{\prime \prime}(I)>0$. We further make the following assumption:

(C) $N \leq b B_{1} / B_{2}$.

It is worth noting that $b \gg 1$, or equivalently, $\gamma \gg \mu+\sigma$, in reality, since the recovery from cholera infection typically occurs in several days [15], whereas the disease conferred immunity lasts several years and the average natural death occurs in tens of years. Similarly, based on realistic data $[6,15,24]$, we have $D_{0} \gg 1$ and thus $B_{1} \gg B_{2}$. Therefore, in practical sense, the assumption (C) does not impose a strong restriction on the total population size $N$. Here we introduce this condition to facilitate our analysis that follows.

Based on assumption (C), we obtain

$$
g^{\prime \prime}(I)=-6 b B_{2} I+2\left(B_{2} N-b B_{1}\right)-2 D_{1} D_{0} \frac{N+b D_{0}}{\left(I+D_{0}\right)^{3}}<0 .
$$


Now we denote $\varphi(I)=f^{\prime}(I)-g^{\prime}(I)$. Then $\varphi(I)$ is increasing since

$$
\varphi^{\prime}(I)=f^{\prime \prime}(I)-g^{\prime \prime}(I)>0
$$

In addition, notice that $\mathcal{R}_{0}=\frac{B_{0}}{A_{0}} N=\frac{g(0)}{f(0)}$. Hence, the following results can be obtained:

(1) If $\mathcal{R}_{0}>1$, then $f(I)$ and $g(I)$ have a unique intersection in $\mathbb{R}_{+}^{2}$.

(2) If $\mathcal{R}_{0}=1$, then there are two possibilities:

(i) If $\varphi(0) \geq 0$, these two curves have no intersection in $\mathbb{R}_{+}^{2}$;

(ii) If $\varphi(0)<0$, there is a unique intersection in $\mathbb{R}_{+}^{2}$.

(3) If $\mathcal{R}_{0}<1$, then there are three possibilities:

(i) If $f(I)>g(I)$ for all $I>0$, then there is no intersection in $\mathbb{R}_{+}^{2}$;

(ii) If there exists $I^{*}>0$ such that $f\left(I^{*}\right)=g\left(I^{*}\right)$ and $f^{\prime}\left(I^{*}\right)=g^{\prime}\left(I^{*}\right)$, then there is a unique intersection in $\mathbb{R}_{+}^{2}$;

(iii) Otherwise, there are two intersections in $\mathbb{R}_{+}^{2}$.

In particular, from cases 3(ii) and 3(iii), we expect that there will be a backward bifurcation under certain conditions, which will make a significant difference to the dynamics of Model One. Below we will provide details of the bifurcation analysis. 


\subsection{Bifurcation analysis}

At a positive equilibrium we have $f(I)=g(I)$ based on equations (3.3.8)-(3.3.10).

Define $g_{1}(I)=B_{2} I^{2}+B_{1} I+B_{0} D_{0}+\frac{D_{1} I}{I+D_{0}}$. Then we have

$$
N=b I+\frac{f(I)}{g_{1}(I)} .
$$

Note that $\mathcal{R}_{0}$ is a positive scalar multiple of $N$. Thus, we pick $N$ as a bifurcation parameter, as the variation of $I$ with respect to $\mathcal{R}_{0}$ can be equivalently represented by the variation of $I$ with respect to $N$ when all other model parameters are fixed. Differentiating (3.4.1) with respect to $\mathrm{N}$ yields

$$
\frac{d I}{d N}=\frac{g_{1}^{2}(I)}{b g_{1}^{2}(I)+f^{\prime}(I) g_{1}(I)-f(I) g_{1}^{\prime}(I)}:=\frac{g_{1}^{2}(I)}{\Phi(I)} .
$$

It is clear that $\frac{d I}{d N}>0$ if and only if $\Phi(I)>0$. We can verify that

$$
\Phi^{\prime}(I)=g_{1}(I)\left(f^{\prime \prime}(I)+2 b g_{1}^{\prime}(I)-\frac{f(I)}{g_{1}(I)} g_{1}^{\prime \prime}(I)\right)=g_{1}(I) \varphi^{\prime}(I)>0 .
$$

Accordingly, if $\Phi(0)<0$, there must be a unique $\tilde{I}$ such that $\Phi(I)<0$ for $0<I<\tilde{I}$ and $\Phi(I)>0$ for $I>\tilde{I}$. Consequently, $\frac{d I}{d \mathcal{R}_{0}}<0$ for $0<I<\tilde{I}$ and $\frac{d I}{d \mathcal{R}_{0}}>0$ for $I>\tilde{I}$. Thus, we find that at $\left(\mathcal{R}_{0}, I\right)=(1,0)$, a backward bifurcation occurs when $\Phi(0)<0$; i.e.,

$$
A_{1}+b B_{0} D_{0}<\frac{A_{0}}{B_{0}}\left(B_{1}+\frac{D_{1}}{D_{0}}\right) .
$$

In contrast, if $\Phi(0) \geq 0,(3.4 .2)$ and (3.4.3) imply that $\frac{d I}{d \mathcal{R}_{0}}>0$ for all $I>0$. Thus, system (3.1.1) has a forward bifurcation that occurs at $\mathcal{R}_{0}=1$ and $I=0$. Therefore, we conclude that $\Phi(0)<0$ is the necessary and sufficient condition under which a 
backward bifurcation occurs.

\subsection{Numerical simulations}

Due to the complexity of model (3.1.1), particularly its high dimension, stability analysis for the equilibria are challenging. Instead, we have conducted extensive numerical simulations to the model with various parameter sets. The results presented below illustrate a few distinct dynamical behaviors of the system.

Figure 3.1a plots $I$ versus $\mathcal{R}_{0}$ for a typical scenario where $\Phi(0)=0.001>0$, as an illustration of the forward bifurcation for the system (3.1.1). In particular, it is highlighted that when $\mathcal{R}_{0}>1$, there exists a unique endemic equilibrium, and when $\mathcal{R}_{0}<1$, there is no positive equilibrium. Figure 3.1b illustrates the backward bifurcation for $(3.1 .1)$ with $\Phi(0)=-0.0026<0$. It is clear to see that there exist two positive equilibrium solutions when $\widehat{\mathcal{R}}_{0}<\mathcal{R}_{0}<1$. Here we use $\left(\widehat{\mathcal{R}}_{0}, \hat{I}\right)$ to denote the turning point, where the two positive equilibrium solutions come together and annihilate each other. The Solid and dashed curves represent the $I$-component of the stable and unstable equilibrium solutions of system (3.1.1), respectively. The value of $\hat{I}$ can be determined from $\Phi(I)=0$, and $\widehat{\mathcal{R}}_{0}$ can be calculated subsequently.

Figure 3.2 plots $I$ versus time for a typical infection curve (time series), where the value of $N$ is chosen such that $\mathcal{R}_{0}=0.86<\widehat{\mathcal{R}}_{0}$. All other parameters take the same values as those in Figure 3.1b. We observe that, after the initial outbreak and a few subsequent oscillations, the infection curve converges to $I=0$, an indication of the stability of the disease-free equilibrium.

Next, we increase the value of $N$ to obtain $\mathcal{R}_{0}=0.976$, while keeping all other parameters unchanged such that $\widehat{\mathcal{R}}_{0}<\mathcal{R}_{0}<1$. In this case, a backward bifurcation occurs, and it is expected that the top branch of the positive equilibrium solution 


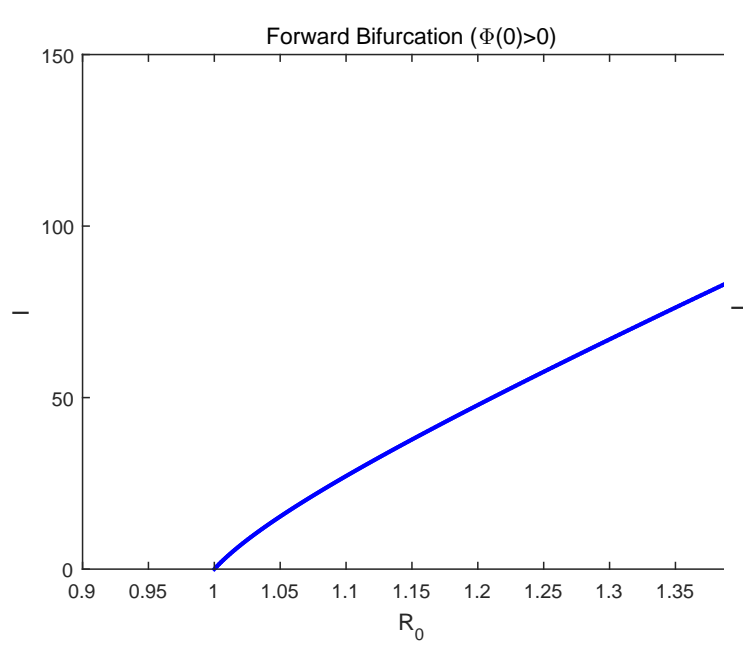

(a)

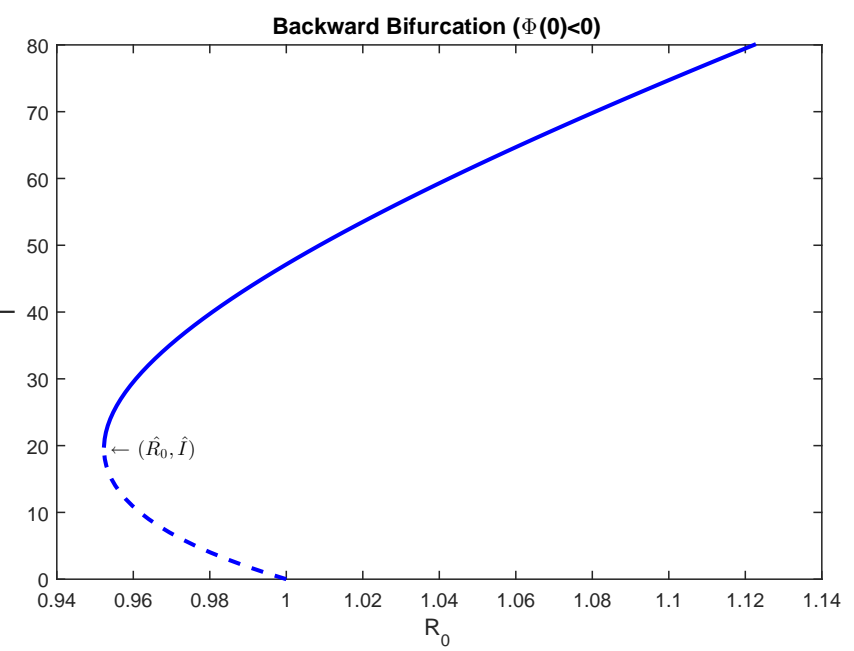

(b)

Figure 3.1 Forward and backward bifurcation

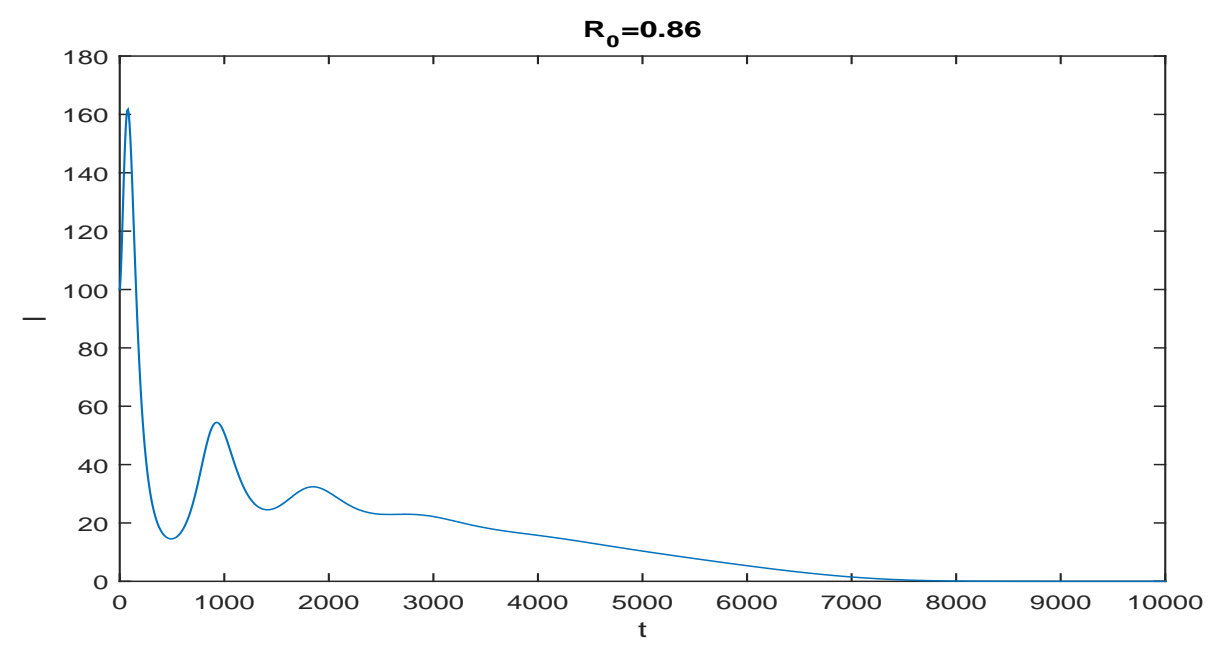

Figure 3.2 The number of infectious humans decays along time 
in Figure $3.1 \mathrm{~b}$ is locally stable. Figure 3.3 shows a phase portrait for $I$ vs. $M$ with a number of different initial conditions, and we clearly observe that all these orbits (including some close to the lower branch of the positive equilibrium solution) converge to the positive equilibrium with $(M, I) \approx(3.1,38)$ located on the top branch. This provides an evidence of the stability of the equilibrium on the upper branch, and the instability of the positive equilibrium on the lower branch (see Figure $3.1 b)$.

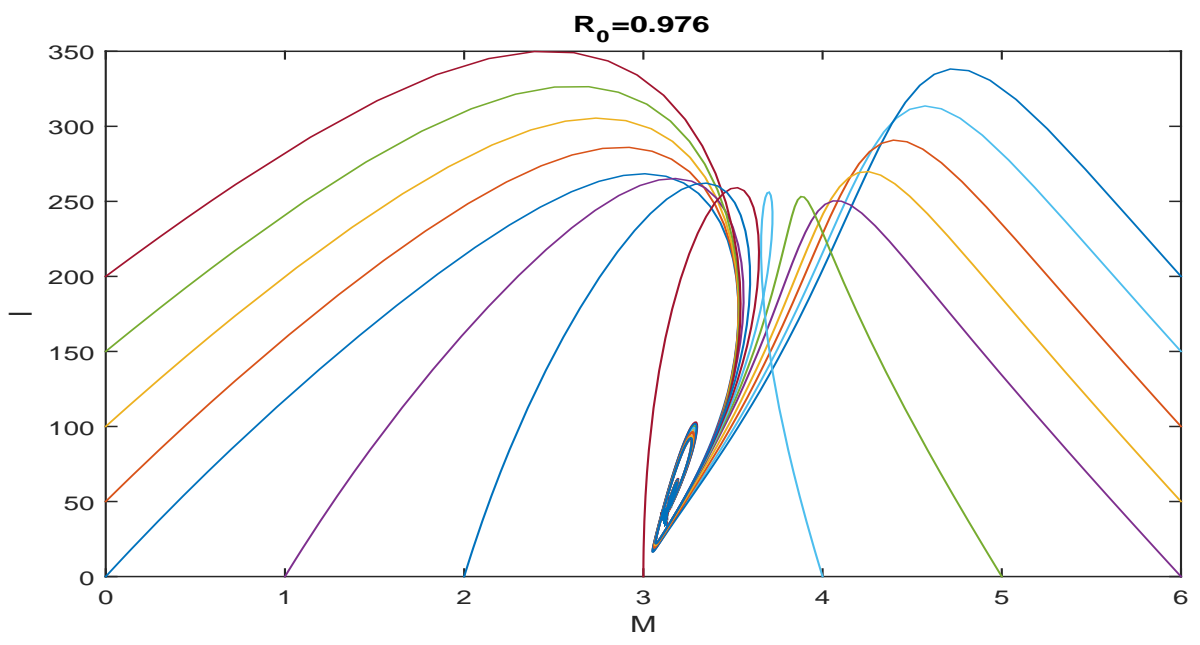

Figure 3.3 Phase portrait for $\mathcal{R}_{0}=0.976$

With $N$ further increased, we obtain $\mathcal{R}_{0}=1.4>1$, and Figure 3.4 shows a phase portrait in the $M-I$ plane for this case. All other parameters take the same values as those under the setting of Figure 3.1a. As can be seen from Figure 3.4, a wide range of initial conditions are used, and all the solution orbits converge to the endemic equilibrium with $(M, I) \approx(3.6,86.6)$, demonstrating the stability of the unique endemic equilibrium. 


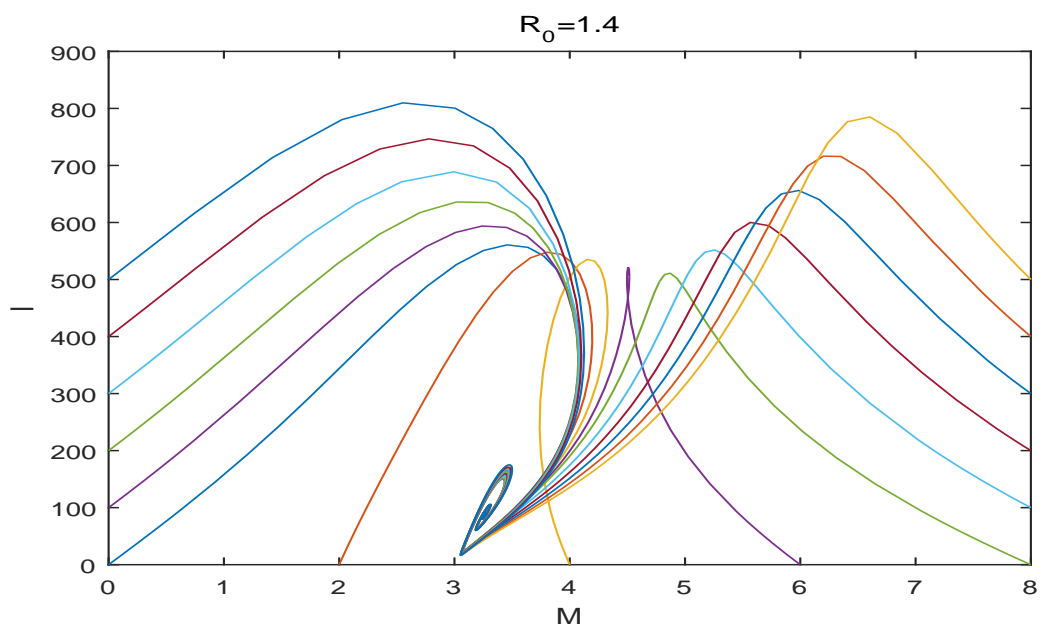

Figure 3.4 Phase portrait for $\mathcal{R}_{0}=1.4$ 


\section{CHAPTER 4}

\section{DISCUSSION}

We have proposed two models to investigate the effect of awareness programs on cholera dynamics. Model One is a five-dimensional system that describes the time evolution of the susceptible, infectious, and recovered human hosts, the bacteria, and the awareness programs. A critical assumption in the first model is that all susceptible individuals have the same probability to access the awareness programs and gain the same level of awareness. The model is then able to explicitly represent the relationship between awareness programs and susceptible individuals in that disease transmission rates and bacteria shedding rate decrease as the number of awareness programs grows.

The dynamical system in Model Two is six-dimensional with two distinct groups (aware and unaware) in the susceptible class. The second model highlights the different levels of infection risk among susceptible individuals due to their awareness/unawareness of the infection (which would result in distinct human behaviors), a practical limitation of any awareness programs. Disease transmission rates within each susceptible class are assumed to be constant, but they take different values across the two susceptible compartments, leading to different degrees of interaction among the human hosts, the environmental pathogen, and the awareness programs.

In the mathematical sense, Model One is much easier to analyze and the results are standard, namely, the disease dynamics are completely determined by the basic reproduction number: if $\mathcal{R}_{0} \leq 1$ then cholera dies out; otherwise the disease persists. 
That is, the first model exhibits regular threshold dynamics. In contrast, Model Two is less trackable, due to the higher dimension of the system and the more complex interaction among compartments. Although rigorous stability analysis has not been resolved for the second model, the mathematical and numerical study has provided a clear picture of its essential, somehow surprising, dynamics. In particular, we have established that Model Two may exhibit both forward and backward bifurcations, a significant difference from the threshold dynamics in Model One. The implication of a backward bifurcation is that reducing $\mathcal{R}_{0}$ below unity is no longer sufficient to eradicate the disease, which brings an additional challenge to the public health administration on cholera control.

The two cholera models are closely related, as they share the same goal of modeling the impact of awareness programs, but from different angles. It is surprising, however, that these two models undergo very different dynamics and would provide different guidelines in cholera control, should they both be used in practical application. It is not our intention to argue which one is more reasonable, as each model emphasizes its own perspective, and all mathematical models are approximations to the reality. A meaningful criterion, in the practical sense, to compare these two models is to verify which one can better fit the observed data, if available. We plan to pursue such a task in our future research. Bifurcation analysis with respect to a controllable awareness-related parameter could be more helpful in understanding the role of health campaigns. We may extend the second model to account for aware and unaware individuals in the susceptible, infectious, and recovered classes [11]. Regardless, our current study provides a modeling framework to investigate the complex cholera dynamics under the impact of awareness programs, and the findings from both models confirm the positive effect of awareness programs 
in lowering the infection risk and reducing the disease prevalence. Our present work also underscores the importance of validating key modeling assumptions and connecting models with realistic data, to guide us in the development and selection of better models toward practical application. 


\section{REFERENCES}

[1] R. M. Anderson, R.M. May, Infectious Diseases of Humans: Dynamics and Control, Oxford University Press, 1991.

[2] J. R. Andrews, S. Basu, Transmission dynamics and control of cholera in Haiti: an epidemic model, Lancet, 377(9773):1248-1255, 2011.

[3] V. Capasso, S. L. Paveri-Fontana, A mathematical model for the 1973 cholera epidemic in the European Mediterranean region. Rev. Epidemiol. Sante, 27(2): $121-132,1979$.

[4] V. Capasso, G. Serio, A generalization of the Kermack-McKendrick deterministic epidemic model, Math. Biosci., 42: 43-61, 1978.

[5] S. Collinson, J. M. Heffernan, Modelling the effects of media during an influenza epidemic, BMC Public Health, 14: 376, 2014.

[6] R. R. Colwell, A global and historical perspective of the genus Vibrio, in The Biology of Vibrios, F.L. Thompson, B. Austin, J. Swings (eds.), ASM Press, Washington DC, 2006.

[7] J. Cui, X. Tao, H. Zhu, A SIS infection model incorporating media coverage, Rocky Mt. J. Math., 38: 1323-1334, 2008.

[8] O. Diekmann, J. A. P. Heesterbeek, J. A. J. Metz, On the definition and the computation of the basic reproduction ratio $R_{0}$ in models for infectious diseases in heterogeneous populations, J. Math. Biol., 28: 365-382, 1990. 
[9] J. Einarsdóttir, A. Passa, G. Gunnlaugsson, Health education and cholera in rural Guinea-Bissau, Int J. Infect. Dis., 5(3): 133-138, 2001.

[10] S. M. Faruque1, G.B. Nair, Vibrio cholerae: Genomics and Molecular Biology, Caister Academic Press, 2008.

[11] S. Funk, E. Gilad, V. A. A. Janse, Endemic disease, awareness, and local behavioural response, J. Theor. Biol., 264: 501-509, 2010.

[12] S. Funk, M. Salathé, V. A. A. Jansen, Modelling the influence of human behaviour on the spread of infectious diseases: a review, J. R. Soc. Interface, $7(50): 1247-1256,2010$.

[13] D. Gao, S. Ruan, An SIS patch model with variable transmission coefficients, Math. Biosci., 232: 110-115, 2011.

[14] Global Task Force on Cholera Control, Cholera outbreak: assessing the outbreak response and improving preparedness, World Health Organization, Geneva, 2004.

[15] D. M. Hartley, J. G. Morris, D. L. Smith, Hyperinfectivity: a critical element in the ability of $V$. cholerae to cause epidemics? PLoS Med., 3: 0063-0069, 2006.

[16] N. Kaur, M. Ghosh, S. S. Bhatia, Modeling and analysis of an SIRS epidemic model with effect of awareness programs by media, Int. J. Math. Comput. Phys. Quant. Eng., 8: 233-239, 2014.

[17] I. Z. Kiss, J. Cassell, M. Recker, P. L. Simon, The impact of information transmission on epidemic outbreaks, Math. Biosci., 225(1): 1-10, 2010. 
[18] M. Kitaoka, S. T. Miyata, D. Unterweger, S. Pukatzki, Antibiotic resistance mechanisms of Vibrio cholerae, J. Med. Microbiol., 60(4): 397-407, 2011.

[19] J. P. LaSalle, The Stability of Dynamical Systems, Regional Conference Series in Applied Mathematics, SIAM, Philadelphia, 1976.

[20] R. Liu, J. Wu, H. Zhu, Media/psychological impact on multiple outbreaks of emerging infectious diseases, Comput. Math. Methods Med., 8: 153-164, 2007.

[21] I. M. Longini, A. Nizam, M. Ali, M. Yunus, N. Shenvi, J. D. Clemens, Controlling endemic cholera with oral vaccines, PLoS Med., 4(11): e336, 2007.

[22] M. E. Lucas, J. L. Deen, L. von Seidlein, et al., Effectiveness of mass oral cholera vaccination in Beira, Mozambique, N. Engl. J. Med., 352(8): 757-767, 2005.

[23] A. K. Misra, A. Sharma, J. B. Shukla, Modeling and analysis of effects of awareness programs by media on the spread of infectious diseases, Math. Comput. Model., 53: 1221-1228, 2011.

[24] Z. Mukandavire, S. Liao, J. Wang, H. Gaff, D. L. Smith, J. G. Morris, Estimating the reproductive numbers for the 2008-2009 cholera outbreaks in Zimbabwe, $P$. Nat. Acad. Sci. USA, 108: 8767-8772, 2011.

[25] E. J. Nelson, J. B. Harris, J. G. Morris, S. B. Calderwood, A. Camilli, Cholera transmission: the host, pathogen and bacteriophage dynamics, Nat. Rev. Microbiol., 7: 693-702, 2009.

[26] D. Posny, J. Wang, Modelling cholera in periodic environments, J. Biol. Dyn., 8(1): 1-19, 2014. 
[27] M.G. Roberts, J.A.P. Heesterbeek, A new method for estimating the effort required to control an infectious disease, Proc. R. Soc. Lond. B 270: 1359-1364, 2003.

[28] Z. Shuai, P. van den Driessche, Global stability of infectious disease models using Lyapunov functions, SIAM J. Appl. Math., 73(4): 1513-1532, 2013.

[29] S. Samanta, S. Rana, A. Sharma, A. K. Misra, J. Chattopadhyay, Effect of awareness programs by media on the epidemic outbreaks: A mathematical model. Appl. Math. Comput., 219(12): 6965-6977, 2013.

[30] H. R. Thieme, Persistence under relaxed point-dissipativity (with application to an endemic model), SIAM J. Math. Anal., 24: 407C435, 1993.

[31] J. P. Tian, J. Wang, Global stability for cholera epidemic models, Math. Biosci., 232(1): 31-41, 2011.

[32] J. H. Tien, D. J. D. Earn, Multiple transmission pathways and disease dynamics in a waterborne pathogen model, B. Math. Biol., 72(6): 1506-1533, 2010.

[33] S. M. Tracht, S. Y. Del Valle, J. M. Hyman, Mathematical modeling of the effectiveness of facemasks in reducing the spread of novel influenza A (H1N1), PLoS ONE, 5(2): e9018, 2010.

[34] P. van den Driessche. J. Watmough, Reproduction numbers and sub-threshold endemic equilibria for compartmental models of disease transmission, Math. Biosci., 180: 29-48, 2002.

[35] J. Wang, S. Liao, A generalized cholera model and epidemic-endemic analysis, J. Biol. Dyn., 6(2): 568-589, 2012. 
[36] X. Wang, J. Wang, Analysis of cholera epidemics with bacterial growth and spatial movement, J. Biol. Dyn., 9(1): 233-261, 2015.

[37] X. Wang, D. Gao, J. Wang, Influence of human behavior on cholera dynamics, Math. Biosci., 267: 41-52, 2015.

[38] J. A. Yorke, W. P. London, Recurrent outbreaks of measles, chickenpox and mumps, II. Systematic differences in contact rates and stochastic effects, Am. J. Epidemiol., 98: 469-482, 1973.

[39] L. Zuo, M. Liu. Effect of awareness programs on the epidemic outbreaks with time delay, Abstr. Appl. Anal., Vol. 2014, Article ID: 940841, 2014. 


\section{VITA}

Yang, Chayu was born in Pengzhou, Sichuan, China, to the parents of Tang, Wenliang and Yang, Yuantong. He is the only child. He attended Hongmiao Elementary School, then Nanyang High School and continued to Pengzhou High School, where he became interested in math, in Pengzhou, Sichuan. After graduation, attended the University of Science and Technology of China where he completed the Bachelors and Master of Science degree in June 2009 and June 2012 in Mathematics, respectively. Yang, Chayu is continuing his education in Applied Mathematics by pursuing a Ph.D. degree at the University of Tennessee at Chattanooga. 